\title{
Previous Trend, Present Prevalence and Integrated Knowledge, Attitude and Practice Towards Malaria and Use of Insecticide-treated Mosquito Net in the Rural Setting of Welkait District, Northwest Ethiopia
}

\section{Tsegay Gebremaryam Yhdego}

Addis Ababa University College of Natural Sciences

\section{Asnake Desalegn Garedew}

Addis Ababa University College of Natural Sciences

Fitsum Tigu Yifat ( $\square$ fitsum.tigu@aau.edu.et )

Addis Ababa University https://orcid.org/0000-0001-9741-0062

\section{Research}

Keywords: Attitude, ITNs, Knowledge, Malaria, Practice, Prevalence, Welkait district, Ethiopia

Posted Date: December 22nd, 2020

DOl: https://doi.org/10.21203/rs.3.rs-48810/v2

License: (c) (1) This work is licensed under a Creative Commons Attribution 4.0 International License.

Read Full License 
1 Previous trend, present prevalence and integrated

2 knowledge, attitude and practice towards malaria and use of

3 insecticide-treated mosquito net in the rural setting of

$4 \quad$ Welkait district, northwest Ethiopia

5 Tsegay Gebremaryam ${ }^{1}$, Asnake Desalegn $^{2}$ and Fitsum Tigu ${ }^{2 *}$

$6{ }^{1}$ Department of Zoological Sciences, College of Natural Sciences, Addis Ababa University,

7 Addis Ababa, Ethiopia.

$8 \quad{ }^{2}$ Department of Microbial, Cellular and Molecular Biology, Division of Microbiology, College

9 of Natural Sciences, Addis Ababa University, Addis Ababa, Ethiopia.

10

$11 *$ Corresponding Author

12 Email address: fitsum.tigu@aau.edu.et (FT)

13

14

15

16

17

18 


\section{Abstract}

20 Background

21 Malaria is still a burden to Ethiopia, especially among the productive ages of the society.

22 Although insecticide-treated mosquito nets (INTs) are widely used by the rural community,

23 malaria remains a leading public health issue. This study aimed to evaluate a 5-year trend,

24 current prevalence and community knowledge, attitude and practice (KAP) towards malaria and

25 use of ITNs as a strategy for prevention of malaria among the rural setting of Welkait district,

26 northwest Ethiopia.

\section{Methods}

28 A cross-sectional study was carried out on 403 households to identify the prevalence rate of 29 malaria. Besides, a 5-year (2015-2019) retrospective data was analyzed. Random sampling 30 technique was used to select representative households among the community. Well-structured 31 questionnaire were used to collect KAP data about malaria and utilization of ITNs. Blood 32 samples were collected and examined for identification of malaria parasites using standard 33 microscopic procedure. SPSS version 22 was used for the analysis of the quantitative data.

\section{Results}

35 Of 36,219 outpatients examined, 7,309 (20.2\%) malaria-positive cases were reported during 36 2015-2019. Overall prevalence of malaria continually declined from 2015-2019. Male slide37 confirmed $(61.4 \%, N=4,485)$ were significantly higher than female $(38.6 \%, N=2,824)(p<$ 38 005). Nineteen (4.7\%) confirmed malaria cases were screened during parasitological survey (12 39 cases of $P$. falciparum and 7 cases of $P$. vivax). Most $78.4 \%$ (316) and $84.1 \%$ (339) of the 40 respondents knew malaria is a communicable and curable disease respectively and 75.6\% (239) 
41 of them associated it with mosquito bit. From $82.4 \%$ (332) respondents who believed malaria is

42 preventable, $81.0 \%$ (269) of respondents considered ITNs as the main prevention method. About

$4364 \%$ (257) of the respondents possessed ITNs, of these, 66.9\% (172) consistently used ITNs

44 during the peak season. Majority 83.6\% (337) of the respondents had a positive attitude towards

45 ITNs.

\section{Conclusions}

47 Although the retrospective data showed a declining trend of malaria in the district, the current

48 prevalence survey revealed significant increase in malaria infection in the locality. This was due

49 to by unsustainable distribution and inconsistent use of ITNs among the population. We

50 recommend the governments and stockholders to give emphasis on full coverage of ITNs and

51 educate the community about ITNs usage.

52 Keywords: Attitude, ITNs, Knowledge, Malaria, Practice, Prevalence, Welkait district, Ethiopia.

\section{Introduction}

54 Malaria is an infectious protozoal disease, transmitted by the bite of parasite-carrier female

55 Anopheles mosquitoes. The causative agent belongs to the genus Plasmodium, that consists of

56 five well known species, namely $P$. falciparum, $P$. vivax, $P$. malariae, $P$. ovale and $P$. knowlesi

57 which commonly cause malaria in humans $[1,2]$. $P$. falciparum and $P$. vivax are the most

58 common and widely distributed species in Ethiopia that account for $60 \%$ and $40 \%$ malaria cases

59 respectively [3]. Among the several Anopheline species that transmit malaria to humans, only

60 Anopheles arabiensis is recognized as primary vector in Ethiopia, while others Anopheles

61 pharoensis, Anopheles funestus and Anopheles nili are considered as the secondary vectors [4].

62 The disease is primarily tropical diseases of most developing countries of the world; which are

63 severely affecting their economy and public health [5]. Overall world malaria cases decreased 
64 from 251 million in 2010 to 228 million in 2018, however, Africa still bears the biggest burden 65 of morbidity with $93 \%$ cases [6]. Despite high morbidity, Africa also recorded the highest 66 absolute reduction of mortality in 2018 (380,000 deaths) compared to 2010 (533,000 deaths) [6].

67 Likewise, the world malaria associated mortality rate declined in 2018 because of the expansion 68 of prevention and control measures. Nevertheless, Sub-Saharan Africa countries are largely 69 affected by malaria infection.

70 Ethiopia is one of the most malaria epidemic-prone countries in Africa with 52.7 million 71 people (68\% of the population) at risk of malaria infection, especially the most productive age 72 groups [7, 8]. Almost $75 \%$ of Ethiopia's mainland is endemic for malaria; furthermore, the 73 interaction of mountainous landscape with variable winds, seasonality of rainfalls, and the 74 presences of ambient temperatures creates diverse micro-climates for malaria transmission [4, 8]. 75 According to the Federal Ministry of Health (FMOH) National Malaria Prevention and Control 76 Strategy, areas below 2,000 m were considered 'malarious' and prevention measures have been 77 in place half a century ago $[9,10]$. Among the most effective malaria control interventions, use 78 of long-lasting insecticidal nets (LLINs) and indoor residual spraying (IRS) of households with 79 insecticide are strongly recommended by WHO $[6,11]$.

80 Several reports indicated consistent and correct utilization of ITNs, particularly the LLINs are 81 proven to reduce the transmission rate up to $90 \%$ [12-14]. Indoor residual spraying is also an 82 extremely effective means of malaria control strategy when properly applied in areas where 83 malaria is epidemic $[11,15]$. In Ethiopia following the WHO recommendation, ITNs have been 84 distributed in all malarious areas free of charge via health workers, volunteers and local 85 administrators. Even though most households owned at least two ITNs per family [10], there is a 86 knowledge gap among the community about consistent and correct use of nets to minimize the 
87 impact of malaria in the country [16]. Full coverage and proper utilization of ITNs are vital for

88 the prevention and control of malaria [17], still there are also limitations in sustainable

89 distribution and timely replacement of nets, seasonality of malaria, and poor knowledge

90 associated with malaria, ITNs and the vector. On top of it, the coverage and utilization of ITNs

91 also differ from region to region [18].

92 The main determinants in the ownership and utilization of ITNs as reviewed by Singh et al.

93 [19] educational level, knowledge of malaria, socio-economic status and parity and community

94 participations were found to be the major factors in different settings. These factors contribute to

95 the low efficacy (60\%) of the available ITNs [19-21]. Bearing in mind, northern Ethiopia, out of

96 the total malaria exposed households, only $74 \%$ of them received at least one ITN [22]. Hence,

97 studying the KAP towards the use of ITNs in such limited resources is vital beside other efforts

98 made to control malaria in the northern region. Furthermore, knowing the past, providing the

99 current prevalence of malaria as well as integrated knowledge, attitude and practice towards

100 utilization of ITNs, completes the protection and control efforts of malaria in the region,

101 especially in the district. Therefore, the current study aimed to analyze the past 5-year (2015-

102 2019) retrospective data, current magnitude of malaria and explore knowledge, attitude and

103 practice towards malaria and use of ITNs as malaria prevention strategy in the rural community

104 of Welkait district, northwest Ethiopia.

105 Methods

\section{Study design}

107 The study was designed to analyze triangulated data sources. A five year (2015-2019) malaria record was extracted from Maygaba Health Center (MHC) and community based cross-sectional 


\section{Study area and population}

112 This study was conducted in Maygaba town of Welkait district, northwest Ethiopia. The 113 Maygaba town is about $929 \mathrm{Km}$ from the capital city-Addis Ababa and $273 \mathrm{Km}$ from Gondar 114 city. It has five rural and one urban villages with an estimated number of 7,039 households. 115 According to the Maygaba town communication office, each village has an average family size of 1164.4 persons per household and a total population of 30,974; of which 15,642 are male and 117 15,332 are female. Three villages (Korarit, Maygaba and Adijamus) with a total population of 11816,781 in 3,814 households were involved in the study. Male and female dwellers were 8,474 $119(50.5 \%)$ and 8,306 (49.5\%), respectively. The population size under each selected village was $1207,266(43.3 \%)$ in Korarit, 4,962 (29.6\%) in Maygaba and 4,553 (27.1\%) in Adijamus. On 121 average, each village consists of 1,271 households and 5,593 population. The villages are 122 characterized by altitudinal range of 677 to 2,755 m.a.s.l. Its mean annual temperature is $22.5^{\circ} \mathrm{C}$, 123 with mean annual minimum and maximum temperature of $15^{\circ} \mathrm{C}$ to $30^{\circ} \mathrm{C}$, respectively. Malaria 124 was frequently reported annually from September to November.

\section{Sample size determination}

126 The sample size was calculated following single population proportion formula, $127 n=\left(\frac{Z \pi}{2}\right)^{2} \times \frac{P(1-P)}{d^{2}}$ reported elsewhere [23]. Assuming that half of the respondents have 128 knowledge on cause and transmission of malaria and use of ITNs with an estimated malaria 129 prevalence rate of $50 \%(p=0.5)$ at $95 \%$ confidence interval $(\mathrm{Z} \alpha / 2=1.96)$ and $5 \%$ of marginal 130 error $(\mathrm{d}=0.05)$. Based on the calculation, value of 384 plus $10 \%$ non-response rate, the total 
131 estimated participants were 422. Actually 403 participants (95.5\% of response rate) were

132 included in both KAP and parasitological studies. A multi-stage cluster random sampling

133 technique was implemented as indicated in Fig 1. A total of $96(422 / 4.4=95.9)$ households were

134 selected using probability proportion to size of households in the selected Gujiles. The

135 proportion of households for each Gujile were divided by the total number of households in a

136 given Gujile to determine a sampling interval for selecting households. A systematic random

137 sampling techniques was used to select every $\mathrm{n}^{\text {th }}$ household and one participant per household

138 was enrolled.

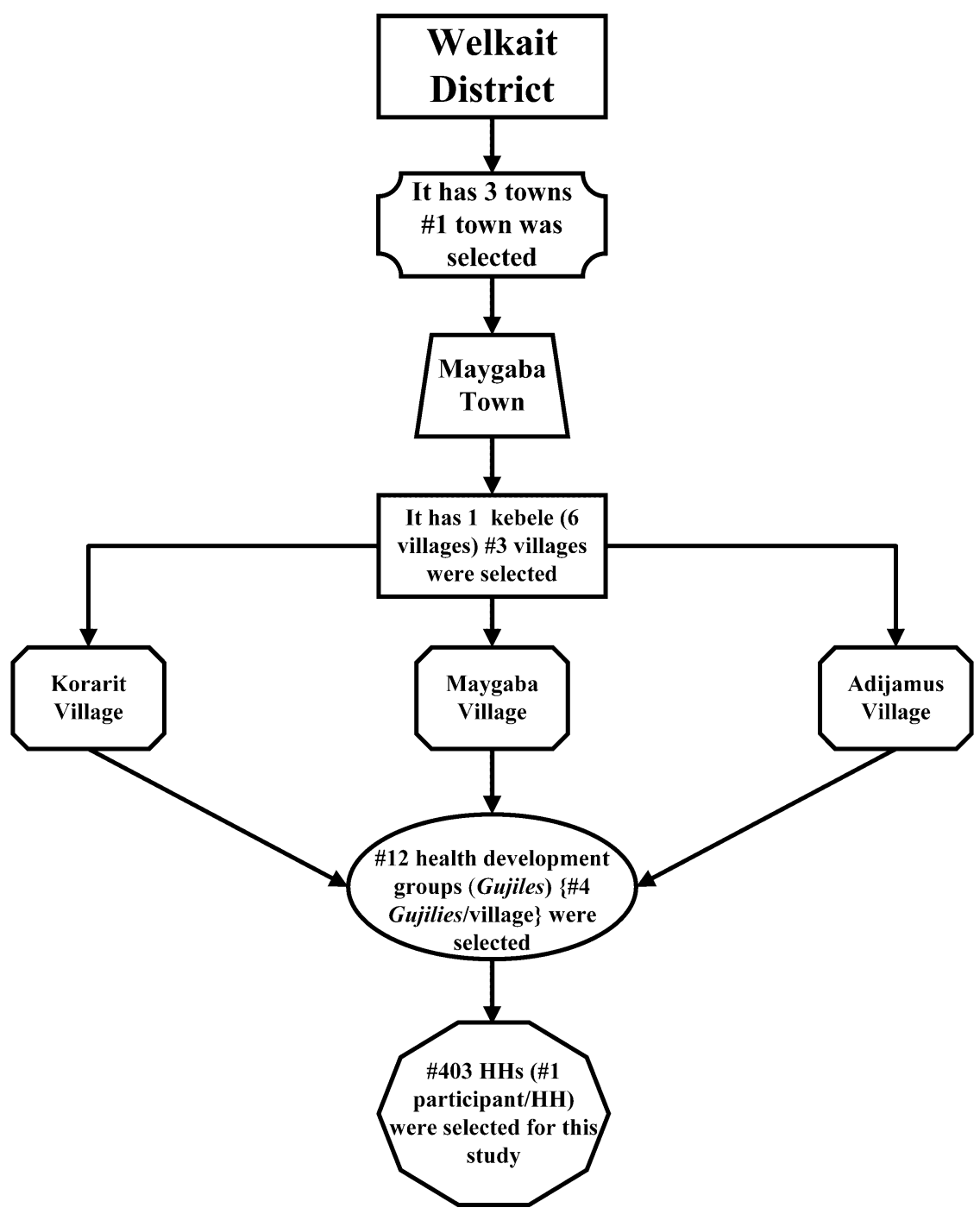

140 Fig 1 Flow chart indicating the multi-stage cluster random sampling technique 


\section{Data collection}

\section{Retrospective health data}

143 The past five years (2015-2019) malaria health examination record data were collected from

144 MHC using the format developed by the principal investigator. The malaria trends in the study

145 area were analyzed by appropriate software.

\section{Cross-sectional study}

147 Well-structured questionnaires containing both close- and open-ended questions about KAP and 148 utilization of ITNs were prepared from earlier studies associated with malaria [16, 24, 25]. The 149 questionnaire was developed in English, translated into Amharic (national language) and 150 checked for correctness of the translation by fluent speakers of both languages. Then questions 151 were pre-tested by preliminary survey in some Gujiles and the content, completeness and 152 suitability towards the target study was validated. Finally, the questionnaire was amended to suit 153 the objectives of the current study and was administered to 403 randomly selected household 154 heads during March to May 2019.

\section{Parasitological examination}

156 Blood samples from 403 households were collected by pricking their finger-tips with the help of 157 laboratory technicians using disposable blood lancet. Thin and thick films were made on the 158 slide and then properly labeled. The thin films were fixed with methanol (100\%) at the site. After 159 air drying in upright position, all the slides were placed in the slide box and carefully transported 160 to the MHC for parasitological examination. Both the thick and thin films were stained with 161 Giemsa (3\%) solution as per the standard microscopic protocol [26]. The presence of malaria 162 parasites in 100 fields were examined from the thick films. The thin films were used for 
163 identification of Plasmodium species. About 5\% of the slides were randomly selected and re-

164 examined by the senior expert in MHC for quality control.

\section{Data analysis}

166 Wholeness and consistency of the data were checked twice and entered into SPSS version 20

167 software (SPSS Inc, Chicago, IL, USA) for statistical analysis. Descriptive statistics (frequencies

168 and percentages) were used to tabulate and describe the cross-sectional data. The Chi-squared

$169\left(\chi^{2}\right)$ test was applied to analyze the retrospective data and determination of association between

170 dependent and independent variables. Statistical significance was defined at $p$-values $<0.05$.

\section{Ethical considerations}

172 The study was ethically approved by the Health Bureau of Welkait district and College of

173 Natural and Computational Sciences Institutional Review Board (CNCS-IRB), Addis Ababa

174 University. Before conducting the investigation, the researcher discussed the study with local

175 administrative body in the study area. All the study population were clearly informed about the 176 purpose of the study and kindly asked to participate in the study. Blood samples were collected

177 by trained staff of MHC and all malaria positive cases were carefully treated according to the 178 national malaria guidelines [27]

\section{Results}

\section{Retrospective analysis}

181 During January 2015 to December 2019, about 36,219 patients have been diagnosed for malaria 182 and out of which $20.2 \%(N=7,309)$ were slide-positive. On average 7,244 malaria-suspected 183 and 1,462 malaria-confirmed cases were tested by MHC every year. Variation in the number of 184 malaria-suspected and -confirmed cases in each year was observed. Despite the variation, the 
185 average monthly malaria prevalence was $1.7 \%$. Generally, the overall prevalence of malaria 186 showed a declining trend during 2015 to 2019, except a slight increase recorded in 2016 (Fig 2a 187 and S1 Table). Regarding the distribution of sex, from the total tested cases, slightly over half $188(54.7 \%, N=19,797)$ of patients were males and $(45.3 \%, N=16,422)$ were females. While from 189 the total slide-confirmed cases, the majority $(61.4 \%, N=4,485)$ of them were males and the rest $190(38.6 \%, N=2,824)$ were females. Looking at the overall male to female ratio of tested and slide191 confirmed cases, mostly males were affected more frequently than females with a ratio of 1.2:1 192 and 1:0.6, respectively. This difference was statistically significant $\left(\chi^{2}=3.923, p<0.05\right)$. 193 Consequently each year, a higher number of malaria positive males were observed than the 194 malaria positive females, however yearly difference in the number of cases was not statistically 195 significant $(p>0.05)$. Comparing the annual overall prevalence of malaria under each sex 196 category, males showed higher (22.7\%) prevalence than females (17.2\%) (Fig 2a and S1 Table). 

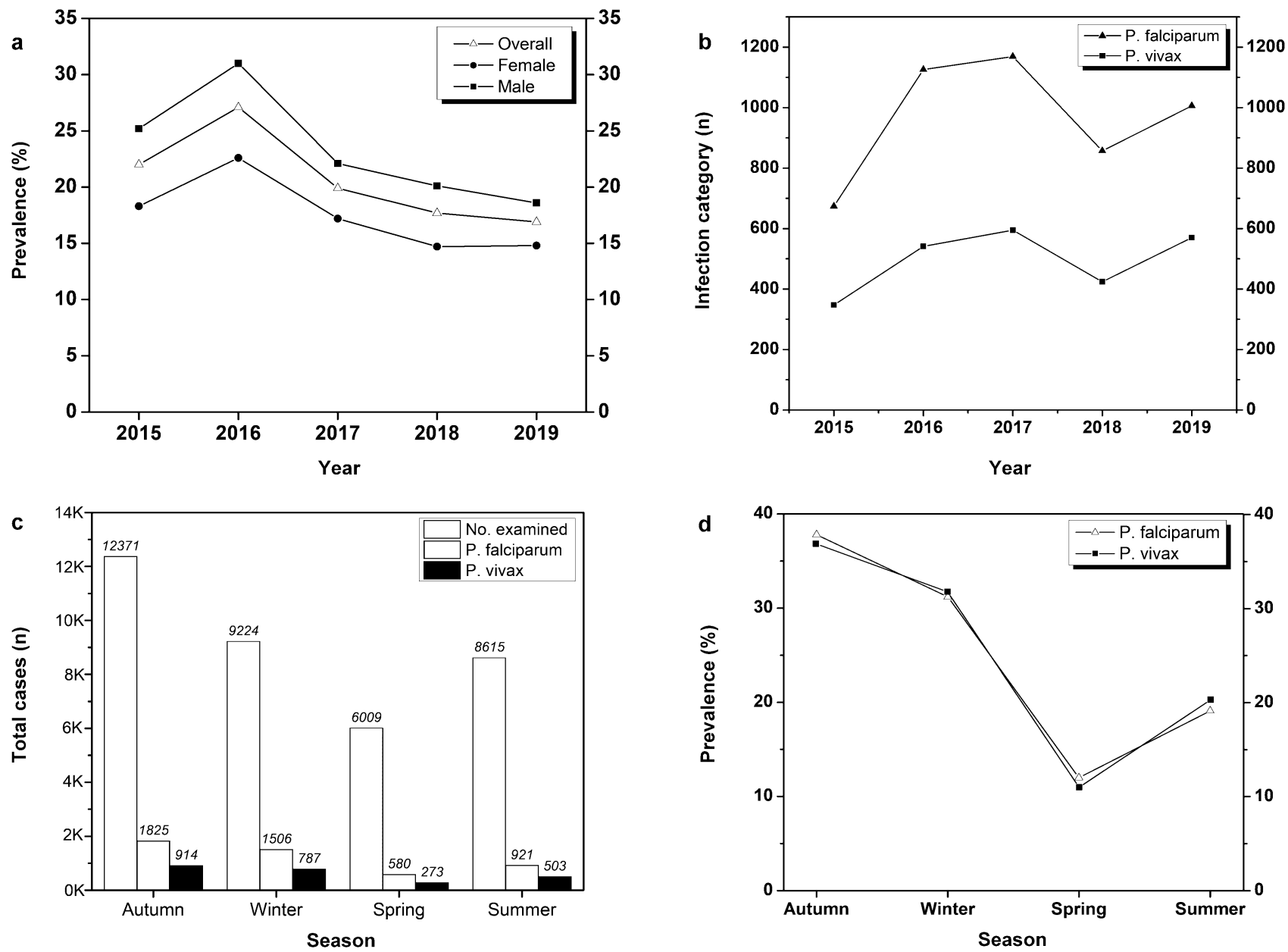

Fig 2 The trend of malaria at MHC, northwest Ethiopia, from 2015-2019 a) Prevalence of malaria b) Side positive cases by infection category c) Seasonal profile of malaria suspected and slide-confirmed cases d) Seasonal prevalence by malaria types

The total number of malaria examined over malaria positive cases showed great variation between seasons. With respect to the number of cases, total cases exhibited the following order: autumn $>$ winter $>$ summer $>$ spring (Figs 2c and d). Majority of suspected as well as infected cases were observed soon after the main rainy season (September-November). A total of 34.2\%

207 of malaria negative and $37.5 \%$ malaria positive cases were reported in this season (S2 Table).

208 While the smallest number of malaria-suspected (16.6\%) and -infected (11.7\%) cases were reported during the small rainy season (March-May). Almost the same malaria-suspected cases 
210 (25.5 and $23.8 \%)$ were observed in dry and heavy rainy seasons respectively (S2 Table).

211 Significantly higher number of slide-confirmed malaria cases (37.5\%) were observed during

212 autumn soon after the heavy rainy season $(p<0.01)$ compared to dry and small rainy seasons.

213 Generally, pairwise comparisons indicates that there are significant inter-seasonal variations $(p<$

$2140.001)$ except between autumn and summer $(p=0.376)$. Since the actual season of malaria in the

215 study area was not clearly defined, it is not possible to calculate the prevalence of malaria for

216 each season. Nevertheless, this imperative result tells us that malaria was observed throughout all

217 the seasons.

218 In terms of infectious category, P. falciparum was the major (66.1\%) contributor to malaria 219 infection in the study area, while $P$. vivax accounted only (33.9\%) of infection (Fig 2b and S1 220 Table). The difference was statistically significant $\left(\chi^{2}=758.8, p<0.001\right)$. Co-infection of $P$. 221 falciparum and $P$. vivax was not reported at all during the 2015-2019 retrospective study. The 222 overall trend of both infections showed variation between years and seasons. Apparently $P$. 223 falciparum was the dominant infection that occurred consistently over $65 \%$ in all study years and 224 seasons (Figs $2 \mathrm{~b}$ and $\mathrm{c}$ ). So, it is not surprising that $P$. falciparum was the most prevalent 225 infection than P. vivax in the past five years at the study site. Despite dominance and prevalence, 226 both infections followed similar patterns towards seasonality (Figs 2c and d). About 37.8, 31.2 227 and 19.1 of $P$. falciparum infection were registered in September-November, December228 February and June-August, respectively (Fig 2d and S2 Table). Similarly, about 36.9, 31.8 and 22920.3 of $P$. vivax cases were also recorded in September-November, December- February and 230 June-August, respectively. Although lower number of $P$. falciparum (12\%) as well as $P$. vivax $231(11 \%)$ cases were reported in the small rainy season than any other seasons, however, 232 statistically significant difference was not noticed $\left(\chi^{2}=5.67, p=0.234\right)$. 


\section{Prevalence of malaria}

234 Only 403 households who provide complete information were considered in blood films 235 collection (Table 1). From which two-hundred and forty-eight (61.5\%) blood donors were male.

236 A total of nineteen slide-positives were obtained, of which twelve cases were from male and 237 seven cases were from female. Nearly half $(48.9 \%)$ of the blood film providers were the most 238 productive age group (18-30 years) of the society with sixteen cases of malaria. While only a few 239 number of participants $(13.9 \%)$ were above 41 -years. The overall prevalence of malaria in the 240 district was $4.7 \%$ with asymptomatic infection category of twelve cases of $P$. falciparum and 241 seven cases of $P$. vivax.

242 Table 1 Socio-demographic characteristics of study population diagnosed for malaria at MHC $(N$ $243=403$ )

\begin{tabular}{|c|c|c|c|c|c|}
\hline Variables & Category & $\begin{array}{l}\text { No examined } \\
(\%)\end{array}$ & $\begin{array}{l}\text { Slide-positive } \\
(\%)\end{array}$ & $\begin{array}{l}P . \\
\text { falciparum }\end{array}$ & P. vivax \\
\hline \multirow[t]{3}{*}{ Sex } & Male & $248(61.5)$ & $12(2.97)$ & 8 & 4 \\
\hline & Female & $155(38.5)$ & $7(1.73)$ & 4 & 3 \\
\hline & Total & 403 (100) & $19(4.7)$ & 12 & 7 \\
\hline \multirow[t]{7}{*}{ Age (year) } & 18-30 (male) & $126(31.3)$ & $10(2.48)$ & 6 & 4 \\
\hline & 18-30 (female) & $71(17.6)$ & $6(1.48)$ & 4 & 2 \\
\hline & 31-40 (male) & $80(19.8)$ & $1(0.25)$ & 1 & 0 \\
\hline & 31-40 (female) & $70(17.4)$ & $1(0.25)$ & 0 & 1 \\
\hline & $>41$ (male) & $42(10.4)$ & $1(0.25)$ & 1 & 0 \\
\hline & $>41$ (female) & $14(3.5)$ & $0(0.0)$ & 0 & 0 \\
\hline & Total & $403(100)$ & $19(4.7)$ & 12 & 7 \\
\hline
\end{tabular}

\section{Participant demographics}

245 During a cross-sectional survey, a total of 422 household heads or their representatives were 246 involved. Out of which nineteen households were excluded from the analysis due to the 
247 incompleteness of the information provided. Four-hundred three participants' information were 248 completed with a response rate of $95.5 \%$. The majority of the respondents were male $(61.5 \%, N$ $249=248$ ). The details of the participants' demographic data are summarized in Table 2. Almost half $250(48.9 \%, N=197)$ of the respondents were in the age category of 18-30 years followed by the $25137.2 \%, N=150$ who belonged to the 31-40 years age group. Majority of the households 252 consisted of greater than five people/household $(62.3 \%, N=251)$; over half $(53.6$ and $54.3 \%)$ of 253 the respondents were illiterate and farmers, respectively.

254 Table 2 Socio-demographic characteristics of study population $(N=403)$

\begin{tabular}{lll}
\hline Variables & Category & Frequency (\%) \\
\hline Sex & Male & $248(61.5)$ \\
Female & $155(38.5)$ \\
Age & $18-30$ & $197(48.9)$ \\
& $31-40$ & $150(37.2)$ \\
& $>41$ & $56(13.9)$ \\
Education & Illiterate & $216(53.6)$ \\
& Elementary & $173(42.9)$ \\
Livelihood & Secondary & $14(3.5)$ \\
& Farmer & $219(54.3)$ \\
& Merchant & $10(2.5)$ \\
Family size (age $<18$ years $)$ & $1-3$ persons & $46(11.4)$ \\
& Student & $114(28.3)$ \\
& Housewife & $57(14.1)$ \\
& Daily laborer & $3(0.7)$ \\
& Korarit & $164(40.7)$ \\
& Maygaba & $147(36.5)$ \\
& & $92(22.8)$ \\
& & 251 \\
& & \\
& & \\
& &
\end{tabular}




$\begin{array}{lll}\text { Livestock ownership } & \text { Yes } & 349 \\ & \text { No } & 54 \\ \text { Ownership \& type of house } & \text { Modern } & 231 \\ & \text { Conventional } & 172\end{array}$

255

256 Knowledge and practice on malaria

257 Majority of the participants $(91.3 \%, N=368)$ living in the study area had awareness of the 258 presence of malaria in their vicinity. Similarly, most of the respondents $(78.4 \%, N=316)$ replied 259 that malaria is a communicable disease, of which $(75.6 \%, N=239)$ knew that malaria is 260 transmitted by mosquitoes. Two-hundred and thirty-nine respondents $(86.6 \%, N=207)$ 261 recognized that night time is suitable for mosquito biting. Most $(82.4 \%, N=332)$ of respondents 262 replied that malaria is a preventable disease. Among the three-hundred and thirty-two 263 respondents, the majority $(81.0 \%, N=269)$ of participants knew that ITNs are the main 264 protection tool for malaria infection. Likewise, a large proportion $(84.1 \%, N=339)$ of the 265 respondents recognized that malaria is treatable if managed earlier; of whom $(82.3 \%, N=279)$ of 266 the participants preferred health centers as ways of treatment (Table 3).

267 Table 3 Malaria related knowledge and practice of the study participants $(N=403)$

\begin{tabular}{lll}
\hline Variables & Category & Frequency (\%) \\
\hline Ever heard about malaria & Yes & $368(91.3)$ \\
& No & $11(2.7)$ \\
Malaria is a communicable disease & I do not know & $24(6.0)$ \\
& No & $316(78.4)$ \\
& I do not know & $64(15.9)$ \\
Cause of malaria $(\mathrm{n}=316)$ & Mosquito bite & $23(5.7)$ \\
& Environmental change & $239(75.6)$ \\
& & $44(13.9)$
\end{tabular}


Lack of environment sanitation $33(10.4)$

When mosquitos bite mostly ( $\mathrm{n}=239)$ Day $15(6.3)$

Night

$207(86.6)$

Any time

$17(7.1)$

Malaria is preventable

Yes

$332(82.4)$

No

$24(6.0)$

I do not know

47 (11.7)

Prevention methods $(\mathrm{n}=332)$

Apply environmental sanitation $9(2.7)$

Use of ITN

$269(81.0)$

Fumigation

$14(4.2)$

Wearing long sleeved clothes

$40(12.1)$

Malaria is treatable

Yes

No

I do not know

43 (10.7)

Ways of treatment $(n=339)$

Traditional healer

$22(6.5)$

Health centers

Buy drug from pharmacy

Others

$8(2.4)$

268

269

\section{Integrated KAP on utilization of ITNs}

270 Majority of the respondents $(75.7 \%, N=305)$ had heard about ITN according to Table 4.

271 Although their sources of information on ITN were varied, most $(73.9 \%, N=227)$ of the

272 respondents obtained information from health workers. With regard to ITNs ownership, about

$27364.0 \%(N=257)$ of the respondents possessed ITNs. Of the two-hundred and fifty-seven ITNs

274 owners, $71.6 \%(N=184)$ of the respondents had one ITN per family, followed by two $15.2 \%(N$

$275=39)$ and three $13.2 \%(N=34)$. On the other hand, from one-hundred and forty-six ITNs non-

276 owners, 56.2\% $(N=82)$ mentioned that they hadn't received ITN, while $43.8 \%(N=64)$ replied

277 that their ITN was worn-out due to wear and tear. Beside the knowledge of respondents towards 
278 ITNs utilization, most of the participants $(83.6 \%, N=337)$ also have a positive attitude towards 279 the benefits of sleeping under ITN. Out of $83.6 \%(N=337)$ respondents, $87 \%(N=292)$ of 280 participants believed that ITN can protect mosquito bites. While the rest of respondents used ITN 281 for other purposes than malaria prevention such as comfortable sleeping and others (Table 4).

282 Out of two-hundred and fifty-seven respondents having ITNs, a considerable number $(67.3 \%$, $283 N=173$ ) of respondents had slept under ITN the last night before the time of interview. A 284 similar figure $(60 \%, N=154)$ had the best experience of utilizing their ITN over 2 years and $28540 \%(N=103)$ of respondents have used on average 1.5 years. Nearly 67\% (172) of the 286 respondents commonly used the ITN during the peak season of malaria. Mostly pregnant women 287 were given priority to use ITN among the family members $(60.3 \%, N=155)$, followed by 288 mother and children under five $(39.7 \%, N=102)$. Despite possession, most of the respondents $289(74 \%, N=190)$ did not re-treated their ITNs with insecticide for the second time. This was due 290 to two main reasons; lack of awareness and lack of insecticide. However, a quarter of the 291 households $(26 \%, N=67)$ were properly utilizing their ITNs with insecticide treatment (Table $2925)$.

293 Table 4 Knowledge and attitude towards the use of ITN among the study population $(N=403)$

\begin{tabular}{lll}
\hline Variables & Category & Frequency (\%) \\
\hline Ever heard about ITN & Yes & $305(75.7)$ \\
& No & $98(24.3)$ \\
Source of information about ITN (n=305) & Mass media (TV \& radio) & $65(21.3)$ \\
& Health workers & $227(73.9)$ \\
& Local leader & $13(4.3)$ \\
ITN possessed & Yes & $257(63.8)$ \\
& No & $146(36.2)$ \\
ITNs per family $(\mathrm{n}=257)$ & One & $184(71.6)$ \\
& Two & $39(15.2)$
\end{tabular}


Three

Reason for ITN non-possession $(n=146) \quad$ Worn-out

Not received

Sleeping under ITN is beneficial

No

What are the benefits of ITN $(n=337)$
Yes

Protect mosquito bite

Comfortable sleep

Others
34 (13.2)

64 (43.8)

82 (56.2)

337 (83.6)

66 (16.4)

$292(86.7)$

$35(10.4)$

$10(3.0)$

295 Discussion

296 The five years retrospective data analysis revealed that the overall prevalence of the malaria was

297 showing a declining trend except a slight increase observed in 2016, this was due to the 298 occurrence of unseasonal rainfall in the study area as the information obtained from the MHC 299 indicates. Although the health system data showed a continual declining of malaria prevalence in 300 the study area, the current prevalence rate obtained from community based parasitological survey 301 do not support this finding, proves that the efforts that have been made by the district as well as 302 the regional health office to control malaria in the study area was not sufficient. This was also 303 further identified by unsustainable distribution and inconsistent use of ITNs observed during the 304 current community based cross-sectional study. Despite the intensification of nationwide malaria 305 interventions such as high coverages of ITN and IRS, improved health services systems and 306 diagnosis of malaria, there is still high incidence of malaria and major discrepancy between ITN 307 ownership and compliance in malaria endemic areas [3]. These has been observed in most 308 settings of sub-Saharan African countries [19]. The rise of malaria prevalence may not be 309 necessarily associated with nationwide or universal coverage of ITN, it might be also due to an 
310 increase in insecticide resistance, inconsistent use of bed nets and under-utilization of other

311 methods of malaria control [28].

312 Table 5 Practices of the study population towards ITN utilization $(N=257)$

\begin{tabular}{lll}
\hline Variables & Category & Frequency (\%) \\
\hline ITN used last night & Yes & $173(67.3)$ \\
Duration of ITN utilization & No & $84(32.7)$ \\
& $1-2$ years & $103(40.1)$ \\
When ITN used mostly & Regularly & $154(59.9)$ \\
& Malaria season & $73(28.4)$ \\
Who mostly used ITN in the family & Mother and children $(<5)$ & $102(39.7)$ \\
& Pregnant woman & $155(60.3)$ \\
Re-treated ITN with insecticide & Yes & $67(26.1)$ \\
& No & $190(73.9)$ \\
If no, what are the reasons $(n=190)$ & Lack of awareness & $89(46.8)$ \\
& Lack of insecticide & $101(53.2)$ \\
\hline
\end{tabular}

313

314 This study revealed that the current prevalence of malaria in the Welkiat district was found to 315 be $4.7 \%$, this finding was much higher than the prevalence reported in other malaria endemic 316 areas of Abeshge (0.25\%) [24] and Shewa Robit town (2.8\%) [25], while lower than other 317 regions of Ethiopia such as Dembia (6.7\%) [29] and Dejen districts (12.4\%) [30] in northwest 318 and east Gojam zones, respectively. Since malaria infestation and incidences depend on different 319 factors such as climate, landscape and altitude of a given area are the factors for the 320 inconsistencies. These differences might also arise from methodological capability and the type 321 of diagnostic tools used, the established malaria control facilities of the areas, the study 322 population and social settings. In contrast, the current prevalence has demonstrated the weakness 
323 of malaria control and prevention efforts of the district. The $4.7 \%$ prevalence rate observed by 324 community based parasitological survey revealed a 3\% increment compared to both monthly $325(1.7 \%)$ and yearly (20.2\%) prevalence calculated from MHC data. Since the household survey 326 was conducted in the small rainy season (March to May), the prevalence might be higher than 327 the current rate if the survey was conducted in the peak season of malaria. Thus, the current 328 result was inconsistent with the finding of Yimer et al. [24] and country-wide reduction of 329 malaria morbidity and mortality report [3].

330 The health record data of this study indicate that significant number of male malaria-confirmed 331 cases were recorded in the MHC compared to the female, suggesting that males were extremely 332 infected by malaria in the study area. The result also coincides with the finding obtained from a 333 parasitological survey of the current study (Table 1). This finding was comparable with a similar 334 study reported in another malaria endemic area of Ethiopia [24]. This is because males' had 335 greater occupational risk of getting the disease than women. Males, mainly 18-40 years old are 336 usually engaged in outdoor activity such as farming particularly, irrigation activity was mostly 337 done during evening up to night in the study area. This was also explained by community based 338 cross-sectional survey revealed that occupation has significant $(p=0.013)$ relationship with 339 knowledge of malaria (Table 6). Furthermore, other behavioral risk factors might increase the 340 risk of mosquito bites among males.

341 Malaria transmission was recorded throughout all the seasons in the study area, and the inter342 seasonal variations were significant $(p<0.001)$. The substantial number of malaria-infected 343 cases were reported shortly after the main rainy season. This is because the existence of cold and 344 cloudy weather conditions during this season create a conducive environment for breeding of 345 mosquitoes [25]. Furthermore, most cultivated crops including maize release pollen grains 
346 following the rainy season that may serve as a food source for mosquito larvae to complete its

347 life cycle. In both retrospective and community based cross-sectional surveys, $P$. falciparum was

348 predominantly found in the study area and become a major (66.1\%) contributor of morbidity and

349 mortality. This is in agreement with the national report $[4,31]$ as well as reports from most other

350 regions of Ethiopia [3, 29].

351 Table 6 Association of knowledge on malaria and ITN utilization with socio-demographic

352 characteristics $(N=403)$

Knowledge on Malaria and Utilization of ITN

\begin{tabular}{llllll}
\hline Characteristics & Poor $(\mathbf{F}, \%)$ & Good $(\mathbf{F}, \%)$ & High $(\mathbf{F}, \%)$ & $\chi^{2}$ & $p$-value \\
\hline Sex & & & & & \\
Male & $75(30.2 \%)$ & $98(39.5 \%)$ & $75(30.2 \%)$ & 0.026 & 0.871 \\
Female & $47(30.3 \%)$ & $63(40.7 \%)$ & $45(29 \%)$ & & \\
Age & & & & & \\
$18-30$ & $58(29.4 \%)$ & $81(41.1 \%)$ & $58(29.4 \%)$ & & \\
$31-40$ & $44(29.3 \%)$ & $62(41.3 \%)$ & $44(29.3 \%)$ & 0.851 & 0.654 \\
$>41$ & $21(37.5 \%)$ & $22(39.3 \%)$ & $13(23.2 \%)$ & &
\end{tabular}

Family size

\begin{tabular}{|c|c|c|c|c|}
\hline $1-3$ person & $15(32.6 \%)$ & $18(39.1 \%)$ & $13(28.3 \%)$ & \\
\hline 4-5 person & $36(34 \%)$ & $42(39.6 \%)$ & $28(26.4 \%)$ & 3.861 \\
\hline >5person & $71(28.3 \%)$ & $100(39.8 \%)$ & $80(31.9 \%)$ & \\
\hline
\end{tabular}

Occupation

$\begin{array}{llllll}\text { Farmer } & 69(31.7 \%) & 86(39.5 \%) & 63(28.9 \%) & 6.219 & 0.013^{*} \\ \text { Non-Farmer } & 52(27.7 \%) & 89(47.3 \%) & 47(25 \%) & & \end{array}$

Education

Illiteracy $\quad 75(34.6 \%) \quad 87(40.3 \%) \quad 54(25 \%)$

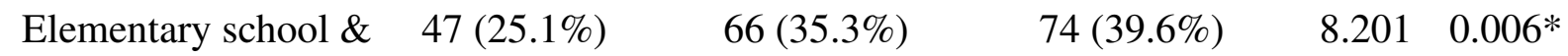
above

Malaria transmission methods
Mosquito bite
$71(29.7 \%)$
$101(42.3 \%)$
$67(28.0 \%)$
$7.612 \quad 0.226$ 
Others

Malaria is treatable

Yes

I do not know

Malaria is preventable

Yes

I do not know
$22(28.6 \%)$

$$
26(33.8 \%)
$$

$29(37.7 \%)$

$102(30.1 \%)$

$$
13(61.9 \%)
$$

$100(30.1 \%)$

$133(40.1 \%)$

$15(62.5 \%)$
$104(30.7 \%)$

$4(19.1 \%)$

$16.980 .002 *$

$99(29.8 \%)$

$4(16.7 \%)$

$0.074 \quad 0.785$
* Significant at $\mathrm{p}<0.05, \mathrm{~F}=$ frequency, $\%=$ percent

The cross-sectional study revealed that the majority $(91.3 \%)$ of the respondents had considered

355 malaria as their major public health problem and over $(75 \%)$ of the respondents also identified

that such disease is transmitted through the biting of mosquitoes. In this study, the awareness of

the respondents was slightly lower than the findings elsewhere [24, 25], while the knowledge of

the transmission of malaria in our study group was in agreement with Alelign and Petros [32].

359 Besides knowledge and awareness on the causes and ways of transmission among the

360 respondents, the knowledge on mosquitoes biting time and prevention measures among the

361 societies are quite important. In this regard, our results indicate that the vast majority $(86.6 \%)$ of

362 the respondents had knowledge on suitable biting time of mosquitoes and about $82.4 \%$ of the

363 respondents also believed that malaria is preventable. Table 6 also shows a significant $(p=$

364 0.002) association between respondents' knowledge on malaria and ITNs utilization and malaria

365 treatability. This finding was comparable with the study conducted in Shewa Robit, northeastern

366 Ethiopia [25], but higher than a similar report in another setting [33].

367 Similarly, about $81.0 \%$ of the respondents mentioned the use of ITNs during sleeping as the 368 main prevention methods from malaria disease. This result supports the respondents' knowledge 369 towards the cause of malaria and its transmission through mosquito biting was quite high even 370 though significant $(p>0.05)$ relationship was not observed (Table 6). The finding was 
371 comparable with other findings elsewhere $[16,24]$, but lower than other reports in Woreta,

372 northwest Ethiopia [32]. The variation may be due to the extent of malaria in the area and socio-

373 cultural differences found in various settings. Although several treatment seeking practices were

374 mentioned by the respondents, nonetheless the majority $(82.3 \%)$ of them preferred health centers

375 for the treatment of malaria disease. This finding was consistent with respondents in other

376 studies elsewhere [24]. The respondents' preference might be due to the availability of health

377 centers than zonal and referral hospitals in the rural settings. Thus, providing malaria related

378 facilities in such centers is an important strategy for the reduction of malaria morbidity and

379 mortality in the district.

380 In addition to assessing the knowledge about malaria vectors, ways of transmission and

381 treatment seeking behaviors of the community, proper knowledge on prevention and control of

382 the malaria as well as the vector are also important to reduce the incidence of the disease among

383 the community. In this regard, combined KAP and ITN utilization among the rural settings are

384 vital since the extent of understanding of the preventive and control measure varies from

385 community to community and among individual households. Thus, taking ITN as a major

386 malaria prevention tool [34], over three fourth of our study groups had enough information about

387 the ITN although their sources were varied. However, the ITNs possession rate was found to be

388 64.0\%, which was exactly consistent with 2016 countrywide coverage [3] but lower than the

389 reports in Benishangul Gumuz (90.9\%) [35], Arsi zone (84.2\%) [21] and Gambella region

$390(81.7 \%)$ [36] in Ethiopia. While higher than the study conducted in other similar studies in Raya

391 Azebo and Harari, Ethiopia [18, 33]. This implies that even in the adjacent regions, there was

392 variation in the ownerships of ITNs. Furthermore, this finding was contrary to the national report

393 that every malarious region had at least two ITNs per household [10]. 
Additionally, a significant number (36.2\%) of the respondents did not possess ITN and the

395 reason reported by the study participants for non-possession of ITN were, did not receive $(56.2 \%)$ and worn-out $(43.8 \%)$. This result indicates that there was an unbalanced and unsustainable distribution of ITN in the district. Therefore, regular monitoring of ITNs ownership by the health extension workers and timely replacements of the worn-out ITNs by the regional health office are important for effective prevention and control of malaria in the district. The efficacy of ITNs depends on how regularly and consistently the ITNs are used and people's perception towards utilization of ITNs $[34,37]$. In our study $83.6 \%$ of households had a positive attitude towards the benefits of sleeping under ITN and also about $87 \%$ of them properly understood its usage. Nevertheless, only $67.3 \%$ of them used ITNs last night at the time of interview. This finding was higher than similar studies in Raya Azebo and Arsi zone, Ethiopia $[21,33]$ but lower than the report from other settings in Ethiopia [18, 24, 38]. Whereas, 16.4\% either had a negative attitude towards use of ITN or were uncertain about their use due to various reasons as mentioned by Tarewa et al [16].

In this study we found that $28.4 \%$ and $67 \%$ of the households used ITN regularly and during peak season of malaria, respectively. Interestingly, priority was given to mothers, children and pregnant women in the family to use ITN. This was a common practice of most communities in Ethiopia $[18,33,38]$. In terms of ITNs utilization experience, almost all the households had been using their ITNs at least for one year and above. This finding was in line with the finding elsewhere [38]. ITN is not equivalent to LLIN that requires the second time treatment with insecticide [34], however only quarter of them treated their ITNs with insecticide for the second time during long usage experiences. Due to great insufficiencies of ITNs/LLINs that limits the 
416 full coverage of all malarious regions in Ethiopia as well as other African regions. The trend of

417 re-treatment of ITN with insecticide and repair has to be encouraged among the society [19, 39].

418 Wiring the triangulated findings in this study, excellent KAP towards malaria and ITNs alone

419 does not grant the reduction of malaria related morbidity and mortality in the study area unless

420 and otherwise consistent usage and sustainable distribution of ITNs among the community has to

421 be practiced regularly. This was simply explained by high prevalence of malaria in the study area

422 obtained via community based parasitological survey and retrospective data analysis.

423 Nevertheless, the parasitological study has to be supported by advanced diagnostic methods such

424 as molecular diagnosis using polymerase chain reaction (PCR) to further confirm the results

425 obtained through microscopic examination. Additionally, it could have been better to design the

426 cross-sectional survey accompanied with direct observation of ITNs usage in the study area.

427 Finally, it is difficult to draw better and reliable conclusions from five years of health recorded

428 data, analyzing as much recorded data as possible may provide more qualified conclusions.

429 However, due to poor recordkeeping at MHC and lack of malaria related follow up data, for

430 instance morbidity and mortality data were missing and limited our study.

\section{Conclusions}

432 In conclusion, the overall prevalence of malaria in the study area was declined during the study 433 period of 2015 to 2019 . The community based parasitological survey discovered nineteen slide-

434 positive cases. Majority of the people were aware of the cause of malaria, communicability, 435 preventability and curability of the disease and identified ITNs as a main prevention method. 436 Although the overall community's KAP and ITNs possession rate is acceptable, this does not 437 elucidate substantial usage, sustainable distribution and timely replacement of ITNs in the study 438 area. This negatively influenced the prevention and control effort of malaria among predisposed 
439 societies of the locality. Therefore, urgent emphasis should be given to full coverage of ITNs,

440 regular monitoring and replacements of old and worn-out ITNs and educate the community

441 towards usage and retreatments of the possessed ITNs by the government and all stockholders

442 are strongly recommended.

\section{Supporting information}

444 S1 Table. Suspected and slide-confirmed annual malaria cases at MHC, from 2015-2019

445 S2 Table. Summary of seasonal cases and prevalence of malaria at MHC during 2015-2019

446 Acknowledgments

447 We gratefully acknowledge Addis Ababa University, School of Graduate Program for the 448 provision of financial support. The authors' also would like to thank College of Natural and 449 Computational Sciences, Institutional Review Board (CNS-IRB) for ethical approval. We are 450 also thankful to Federal Minister of Education School of Biological Sciences and Biotechnology, 451 MHC and laboratory technicians and study participants' for their valuable information.

452

453 Ethics approval and consent to participate

454 The study was ethically approved by the Health Bureau of Welkait district and College of 455 Natural Science Institutional Review Board (CNS-IRB), Addis Ababa University. Before 456 conducting the investigation, the researcher discussed the study with local administrative bodies

457 in the study area. All the study population were clearly informed about the purpose of the study 458 and kindly asked to participate in the study. Prior to administering the questionnaires and blood 459 sample collection, all participants agreed and signed the consent form. Blood samples were 
460 collected by trained staff of MHC and all malaria positive cases were carefully treated according

461 to the national malaria guidelines.

\section{Data Availability Statement}

463 All relevant data are within the paper and its Supporting Information files.

464 Competing interests

465 The authors declare that they have no competing interests

466 Funding

467 No specific funding was obtained for this particular study

468 Authors' contributions

469 All authors contributed to the study conception and design. Material preparation and data

470 collection were performed by TG. Statistical analysis and interpretation were performed by TG,

471 AD and FT. The first draft of the manuscript was written by FT and all authors commented on

472 previous version of the manuscript. All authors read and approved the final manuscript.

473 


\section{References}

475 1. Singh S. Current scenario of control of malaria. Trop Parasitol. 2011 Jul1;(2):52-3.

476 2. Singh B, Kim Sung L, Matusop A, Radhakrishnan A, Shamsul SS, Cox-Singh J, Thomas A, 477 Conway DJ. A large focus of naturally acquired Plasmodium knowlesi infections in human 478 beings. Lancet. 2004 Mar 27;363(9414):1017-24.

479 3. Taffese HS, Hemming-Schroeder E, Koepfli C, Tesfaye G, Lee M-c, Kazura J, et al. Malaria 480 epidemiology and interventions in Ethiopia from 2001 to 2016. Infect Dis Poverty. 2018 Nov $481 \quad 5 ; 7(1): 103$.

482 4. Presedent's Malaria Initative Ethiopia. Malaria Operational Plan FY. Ethiopia: PMI; 2019.

483 5. Girum T, Shumbej T, Shewangizaw M. Burden of malaria in Ethiopia, 2000-2016: findings 484 from the Global Health Estimates 2016. Trop Dis Travel Med Vaccines. 2019 Jul 12;5:11.

485 6. World health organization. World malaria report 2019. Geneva: WHO; 2019.

486 7. Alemu MB, Asnake MA, Lemma MY, Melak MF, Yenit MK. Utilization of insecticide 487 treated bed net and associated factors among households of Kola Diba town, North Gondar, 488 Amhara region, Ethiopia. BMC Res Notes. 2018 Aug 13;11(1):575.

489 8. Bihonegn T, Fentahun S. Review on Malaria and Antimalarial Activity of Vernonia 490 Amygdalina in Ethiopia: A Review Article. JHMN. 2019; 60:1-15.

491 9. Chibsa S. Malaria Vector Control Efforts and Challenges in Ethiopia. In $4^{\text {th }}$ WIN Meeting; 2007 Oct 24-26; Basel, Switzerland, 2007. p. 1-35. 
10. Federal Democratic Republic of Ethiopia Ministry of Health. Malaria Prevention \& Control Program Ethiopia: FMOH. http://www.moh.gov.et/ejcc/en (2020). Accessed 4 May 2020.

11. World health organiztion. Indoor residual spraying: an operational manual for indoor residual spraying (IRS) for malaria transmission control and elimination. Geneva: WHO; 2015.

497 12. Belay M, Deressa W. Use of insecticide treated bets by pregnant women and associated 498 factors in a pre-dominantly rural population of northern Ethiopia. Trop Med Int Health. 2008; $499 \quad$ Oct 8; 13(10):1303-1313.

13. Baume CA, Marin MC. Gains in awareness, ownership and use of insecticide-treated nets in $501 \quad$ Nigeria, Senegal, Uganda and Zambia. Malar J. 2008 Aug 7;7:153.

14. Bejon P, Ogada E, Peshu N, Marsh K. Interactions between age and ITN use determine the 503 risk of febrile malaria in children. PLoS One. 2009 Dec 23;4(12): e8321.

504 15. Correa APSA, Galardo AKR, Lima LA, Camara DCP, Muller JN, Barroso JFS, et al. 505 Efficacy of insecticides used in indoor residual spraying for malaria control: an experimental trial on various surfaces in a "test house". Malar J. 2019 Oct;18(1):345.

507 16. Taremwa IM, Ashaba S, Adrama HO, Ayebazibwe C, Omoding D, Kemeza I, et al. 508 Knowledge, attitude and behaviour towards the use of insecticide treated mosquito nets 509 among pregnant women and children in rural Southwestern Uganda. BMC Public Health. 5102017 Oct $10 ; 17(1): 794$.

511 17. Sexton AR. Best practices for an insecticide-treated bed net distribution programme in sub$512 \quad$ Saharan eastern Africa. Malar J. 2011 Jun 8;10:157. 
513 18. Teklemariam Z, Awoke A, Dessie Y, Weldegebreal F. Ownership and utilization of 514 insecticide treated nets for malaria control in Harari National Regional State Estern Ethiopia. $515 \quad$ Pan Afri Med J. 2015 May 25;21:52.

516 19. Singh M, Brown G, Rogerson SJ. Ownership and use of insecticide-treated nets during 517 pregnancy in sub-Saharan Africa: a review. Malar J. 2013 Aug 1;12:268.

518 20. Kanmiki EW, Awoonor-Williams JK, Phillips JF, Kachur SP, Achana SF, Akazili J, et al. 519 Socio-economic and demographic disparities in ownership and use of insecticide-treated bed 520 nets for preventing malaria among rural reproductive-aged women in northern Ghana. PLoS $521 \quad$ One. 2019 Jan 29;14(1):e0211365.

522 21. Ragasa AS, Wodajo LT, Demissie HF. Proper utilization of long lasting treated net and 523 associated factors at Zuway Dugda district, Arsi zone, Ethiopia. J Public Health Epidemiol. $524 \quad 2019 ; 11: 13-24$.

525 22. Tomas Z. Knowledge, Attitude and Practice (KAP) about Insecticide Treated Net (ITNs) 526 usage against Malaria in Kolla Tembien district, Tigray, Ethiopia [MSc Thesis]. Mekelle $527 \quad$ University; 2011.

528 23. Charan J, Biswas T. How to calculate sample size for different study designs in medical 529 research. Indian J Psychol Med. 2013 Apr;35(2):121-6.

530 24. Yimer F, Animut A, Erko B, Mamo H. Past five-year trend, current prevalence and 531 household knowledge, attitude and practice of malaria in Abeshge, south-central Ethiopia. $532 \quad$ Malar J. 2015 Jun 3;14:230. 
533 25. Abate A, Degarege A, Erko B. Community knowledge, attitude and practice about malaria in 534 a low endemic setting of Shewa Robit Town, northeastern Ethiopia. BMC Public Health. $535 \quad 2013$ Apr 8;13:312.

26. Garcia L. Diagnostic Medical Parasitology. 4th ed. Washington: ASM press; 2001.

537 27. Federal Democratic Republic of Ethiopia Ministry of Health. National Malaria Guidelines. $538 \quad$ Addis Ababa: FMOH; 2012.

28. Zamawe COF, Nakamura K, Shibanuma A, Jimba M. The effectiveness of a nationwide universal coverage campaign of insecticide-treated bed nets on childhood malaria in Malawi. Malar J. 2016 Oct 18;15:505.

29. Fekadu M, Yenit MK, Lakew AM. The prevalence of asymptomatic malaria parasitemia and 543 associated factors among adults in Dembia district, northwest Ethiopia, 2017. Arch Public

30. Kassie A, Wale M, Fekensa T. Assessment of insecticide treated bed net possession, proper utilization and the prevalence of malaria, in Dejen Woreda, East Gojam Zone, Ethiopia. J Health. 2018 Dec 20;76:74.

31. Federal Democratic Republic of Ethiopia Ministry of Health. An Epidemiological Profile of 549 Malaria in Ethiopia. National Malaria Control Team \& Ethiopian Public Health Institute. Addis Ababa: FMOH; 2014. preventive measures in Woreta town, Northwest Ethiopia. BMC Res Notes. 2018 Jul $18 ; 11(1): 491$. 
554 33. Tesfay K, Yohannes M, Mardu F, Berhe B, Negash H. Assessment of community 555 knowledge, practice, and determinants of malaria case households in the rural area of Raya 556 Azebo district, Northern Ethiopia, 2017. PLoS ONE. 2019 Oct 15;14(10):e0222427.

557 34. World health organization. Malaria prevention through insecticide treated nets. Geneva: 558 WHO; 2016.

559 35. Duguma A, Woldemichael K, Dejene T, Agajie M. Assessment of Insecticide Treated Bed 560 Net Use and Factors Affecting Its Use For Prevention of Malaria Among Pawe Woreda 561 Community, Benishangul Gumuz, North-West, Ethiopia. IJSRC. 2018;6:13-22.

562 36. Watiro AH, Awoke W. Insecticide-treated net ownership and utilization and factors that 563 influence their use in Itang, Gambella region, Ethiopia: cross-sectional study. Risk Manag $564 \quad$ Healthc P. 2016 May 31;9:101-12.

565 37. Biadgilign S, Reda A, Kedir H. Determinants of Ownership and Utilization of Insecticide566 Treated Bed Nets for Malaria Control in Eastern Ethiopia. J Trop Med. 2012;2012:235015.

567 38. Berkessa T, Oljira D, Tesfa B. Insecticide treated nets use and its determinants among 568 settlers of Southwest Ethiopia. BMC Public Health. 2016 Feb 1;16:106.

569 39. Zewde A, Irish S, Woyessa A, Wuletaw Y, Nahusenay H, Abdelmenan S, et al. Knowledge 570 and perception towards net care and repair practice in Ethiopia. Malar J. 2017 Oct 2;16:396. 


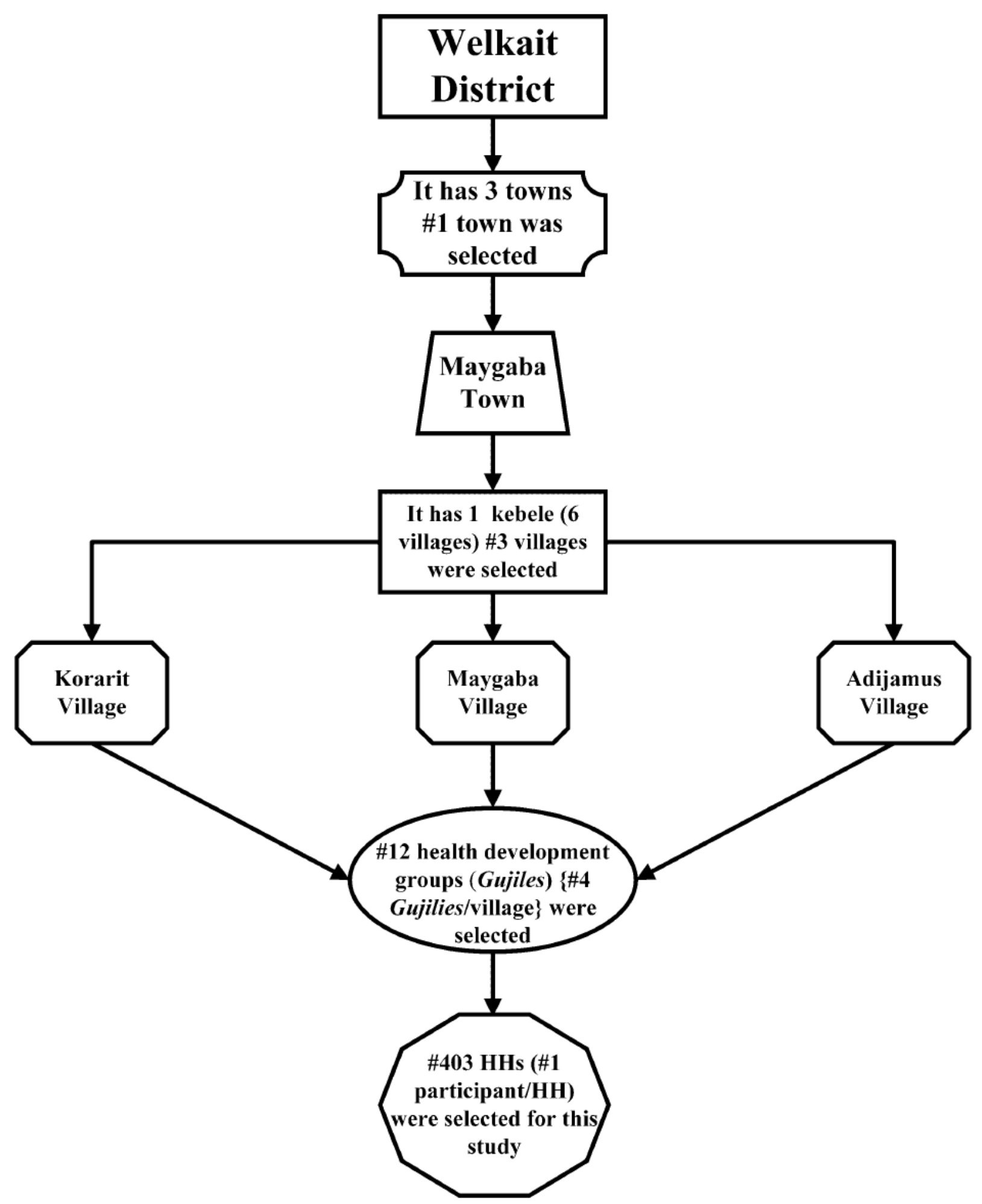

Figure 1

Flow chart indicating the multi-stage cluster random sampling technique 

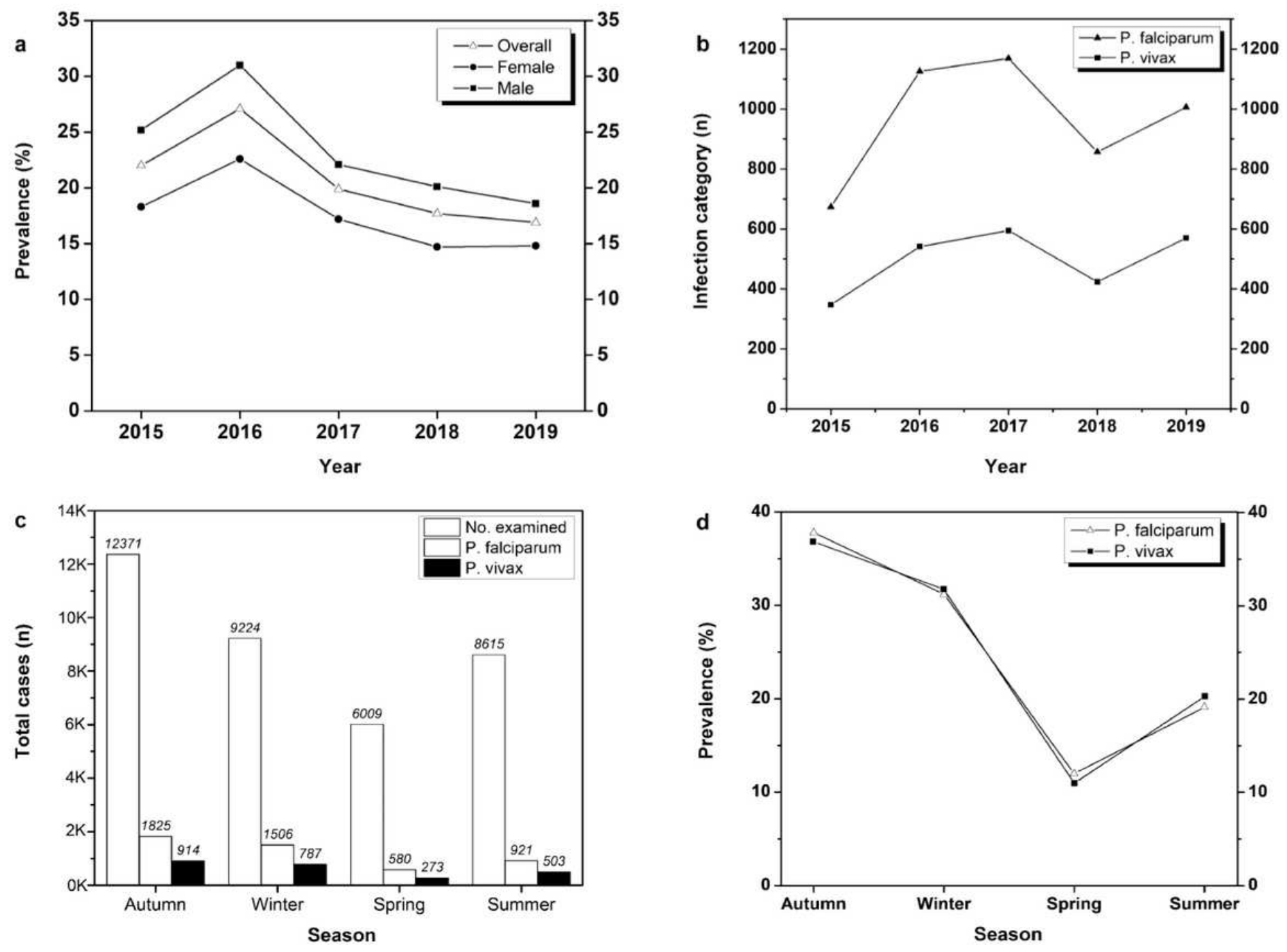

\section{Figure 2}

The trend of malaria at MHC, northwest Ethiopia, from 2015-2019 a) Prevalence of malaria b) Side positive cases by infection category c) Seasonal profile of malaria suspected and slide-confirmed cases d) Seasonal prevalence by malaria types

\section{Supplementary Files}

This is a list of supplementary files associated with this preprint. Click to download.

- S1Table.pdf

- S2Table.pdf 